\title{
Exploration of upper limits of task complexity in concept identification of males and females in individual and social conditions
}

\author{
AARON WOLFGANG ${ }^{2}$, BEHAVIORAL SCIENCE LABORATORY, RADIATION THERAPY SECTION
} WALTER REED GENERAL HOSPITAL, WASHINGTON, D. C.

This study explored limits of male and female Ss in abstracting relevant information in concept identification (CI) problems when learning alone or in pairs. CI was least difficult in free interaction where $S$ s were free to verbalize their hypotheses. Females' concept learning improved with increased irrelevant information whereas males' performance worsened. Ss showed evidence of learning at both complexity levels indicating limits were not reached.

The primary aim of this study was to explore limits of male and female Ss' capacity to abstract relevant $(R)$ information in concept identification (CI) problems of high levels of complexity in individual and social conditions. It has been clearly established both theoretically (Bourne \& Restle, 1959) and experimentally that systematic increases in the number of irrelevant (I) bits (binary stimulus dimensions) of information increased task complexity. In the vast majority of studies, the maximum number of $I$ bits of information introduced in two-choice CI problems has been 5 (i.e., 64 possible stimuli to categorize); in this study the maximum was expanded to 6 (128 stimuli) and 7 bits (256 possible stimuli). Perhaps 7 would be the magic number (Miller, 1956) for approaching limits in CI.

There has been little research dealing with the social aspects of $\mathrm{CI}$, where male or female Ss are free to discuss their hypotheses. In a recent study (Wolfgang, 1967), it was found that two-person male groups free to communicate showed only a slight deterioration in performance even on the most complex problems (5 I bits). In contrast, Individuals and two-person groups where communication was restricted showed progressive decrement on CI problems of increasing complexity. Considering these findings, it was hypothestzed that pairs of Ss free to communicate would make fewer errors in solving CI problems of high complexity than individuals and Ss in the restricted interaction group, and would be least Iikely to approach limits in CI.

Method

One hundred twenty students from introductory sociology classes participated in a 2 by 2 by 2 by 3 factorial design which included two levels of task complexity (6 and 7 I bits of information), two problems (form and number as relevant dimensions), two sex groups, and three interaction conditions where Ss performed with a partner or alone. In the free interaction group (FI), Ss were instructed to reach a mutually agreeable decision and register only one decision per trial; in the restricted interaction group (RI), no discussion was permitted beyond Ss stating and registering their individual decisions by depressing one of the two response keys; and in the third condition, Ss performed alone (A).

A detailed description of apparatus and procedure is given elsewhere (Wolfgang, 1967). In brief, Ss' task was to categorize a series of geometric patterns presented on the screen in accordance with a $\mathbf{R}$ binary stimulus dimension. There was always one bit of $R$ information accompanied by 6 or 7 I bits. Results and Discussion

An analysis of variance on errors disclosed nonsignificant main effects for sex, complexity, and problems ( $p>05$ ) with interaction levels (IL) approaching significance $(F=2.99, d f=2 / 48, p<.10)$. The significant interactions were IL by Problems $(F=4.19, \mathrm{df}=2 / 48, \mathrm{p}<.025)$ and Sex by Complexdty ( $F=5.67, \mathrm{df}=1 / 48, \mathrm{p}<.025$ ).

Mean errors were $39.75,18.46$, and 38.50 for Ss in the A, FI, and RI conditions, respectively. Duncan's test showed, as expected, that Ss in the FI condition made significantly fewer errors than those in the $A$ and RI conditions ( $p<.025$ ), which confirms previous findings (Wolfgang, 1967). Contrary to previous results, learning was not poorest in the $R I$ group. When $\mathrm{Ss}$ in the $\mathrm{RI}$ group reached criterion, they were asked to independently explain the solution. In 9 out of 24 groups (37\%) one of the Ss in the pair who had reached criterion was unable to explain the solution. In an earlier study (Wolfgang, 1965), where CI problems of lesser complexity were used, Ss imitated in only 4 out of 24 (16\%) instances. Thus, with increased task complexity there was more imitation learning resulting in a reduction of overall error scores in the RI condition.

Subsequent analysis of the IL by Problems interaction indicated that individuals made significantly more errors when sorting for number than form $(p<.005)$ which is consistent with the findings of Heidbreder (1949) and Grant (1951), but inconsistent with Wolfgang, Pishkin, \& Lundy (1962). Heidbreder (1945) hypothesized that Ss have more difficulty with number than form because number requires a more analytical approach whereas forms are more directly perceived. However, her hypothesis does not explain why form and number were of equal difficulty in 


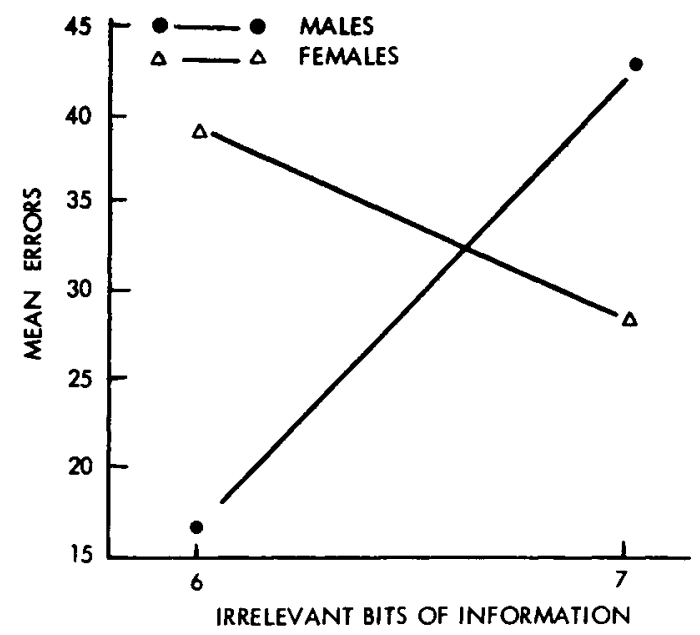

Fig. 1. Mean errors of males and females as a function of number of irrelevant bits of information.

social conditions. Perhaps with pairs of Ss more alternative hypotheses were available and the probability of perseverating on an incorrect hypothesis was reduced. Out of the nine RI groups where imitation learning occurred, $S s$ in seven of those groups imitated their partner's solution when number was relevant.

When an analysis was performed with Duncan's test on the Sex by Problems interaction $(F=3.32$, $\mathrm{df}=1 / 48, \mathrm{p}<.10)$ results showed form and number were of equal difficulty for males, whereas females produced significantly more errors $(p<.05)$ on number $(X=47.41)$ than form concepts $(X=21.25)$. That females found number concepts more difficult is conslstent with past results in problem solving where females perform consistently poorer than males on numerical or arithmetical reasoning problems.

The significant Complexity by Sex interaction indicated that females showed improvement in concept learning with increased irrelevant information. For males the results were reversed (see Fig. 1) which was consistent with past studies. That females' concept learning was not retarded with increased irrelevant information contradicted findings primarily with males, and violated one of the basic assumptions of the mathematical theory of CI (Bourne \& Restle, 1959), which was based mainly on male Ss and was supported by male data.

Figure 1 shows that error differences between males and females are most pronounced on problems with 6 I bits, with females making over 2-1/2 times as many errors as males. It was noted that error scores for males on problems with 5 I bits under similar conditions in a previous study (Wolfgang, 1967) were not appreciably different from those with 6 I bits in this study, indicating that the additional bit of I Information, such as 'background" (white and green), had little effect on learning rates of males. Thus, the results suggest that irrelevant background cues were attended to less by males than females. This finding supports studies reported by Wapner \& Werner (1965) where consistent sex differences on perceptual tests were found, with females being more affected than males by the surrounding field in their perceptions of simple geometric patterns.

In conclusion, overall results suggest that the most optimal condition for CI was in the FI condition where Ss were free to verbalize their hypotheses, and that caution should be exercised in attempting to generalize findings and theory based on males to females. That Ss in all conditions continued to show evidence of learning even on problems with 7 I bits indicated that Miller's (1956) magical number 7 may have to be revised upward in CI.

\section{References}

BOURNE, L. E., JR., \& RESTLE, F. Mathematical theory of concept identification. Psychol. Rev., 1959, 66, 278-296.

GRANT, D. A. Perceptual versus analytical responses to the number concept of a Weigl-type card sorting test. J. exp. Psychol., 1951, 41, 23-29.

HEIDBREDER, EDNA. Toward a dynamic psychology of cognition. Psychol. Rev., 1945, 52, 1-22.

HEIDBREDER, EDNA. The attainment of concepts: VII. Conceptual achievements during card-sorting. J. Psychoh, 1949, 27, 3-39.

MILLER, G. A. The magical number seven, plus or minus two: some limits on our capacity for processing information. Psychol Rev., 1956, 63, 81-97.

WAPNER, S., \& WERNER, H. (Eds.) The body percept. New York: Random House, 1965, Pp. 40-41.

WOLFGANG, A. Effects of social cues and task complexity in concept identification. J. educ. Psychol, 1967, 58, 36-40.

WOLFGANG, A. Learning in concept identification as a function of social cues and complexity in a free social interaction setting. Doctoral dissertation, University of Oklahoma, 1965.

WOLFGANG, A., PISHKIN, V., \& LUNDY, R. M. Anxiety and misinformation feedback in concept identification. Percept. mot. Skills, 1962, 14, 135-143.

Notes

1. This research was supported by the Veterans Administration Medical Research Program (8200 funds) and was done while the author was at the VA Hospital and University of Oklahoma Medical Center, Oklahoma City. Special thanks to Lewis M. Jones for valuable assistance in collecting data.

2. Also at the Walter Reed Army Institute of Research, Neuropsychiatry Division. 